\title{
Video Article \\ Colon Ascendens Stent Peritonitis (CASP) - a Standardized Model for Polymicrobial Abdominal Sepsis
}

\author{
Tobias Traeger ${ }^{*}$, Pia Koerner* ${ }^{*}$, Wolfram Kessler ${ }^{1}$, Katharina Cziupka ${ }^{1}$, Stephan Diedrich ${ }^{1}$, Alexandra Busemann ${ }^{1}$, Claus-Dieter Heidecke ${ }^{1}$, \\ Stefan Maier ${ }^{1}$ \\ ${ }^{1}$ Department of Surgery, University of Greifswald \\ *These authors contributed equally
}

Correspondence to: Tobias Traeger at traeger@uni-greifswald.de

URL: https://www.jove.com/video/2299

DOI: doi:10.3791/2299

Keywords: Immunology, Issue 46, sepsis model, sepsis, peritonitis, mice, surgery, CASP

Date Published: $12 / 18 / 2010$

Citation: Traeger, T., Koerner, P., Kessler, W., Cziupka, K., Diedrich, S., Busemann, A., Heidecke, C.D., Maier, S. Colon Ascendens Stent Peritonitis (CASP) - a Standardized Model for Polymicrobial Abdominal Sepsis. J. Vis. Exp. (46), e2299, doi:10.3791/2299 (2010).

\section{Abstract}

Sepsis remains a persistent problem on intensive care units all over the world. Understanding the complex mechanisms of sepsis is the precondition for establishing new therapeutic approaches in this field. Therefore, animal models are required that are able to closely mimic the human disease and also sufficiently deal with scientific questions. The Colon Ascendens Stent Peritonitis (CASP) is a highly standardized model for polymicrobial abdominal sepsis in rodents. In this model, a small stent is surgically inserted into the ascending colon of mice or rats leading to a continuous leakage of intestinal bacteria into the peritoneal cavity. The procedure results in peritonitis, systemic bacteraemia, organ infection by gut bacteria, and systemic but also local release of several pro- and anti-inflammatory cytokines. The lethality of CASP can be controlled by the diameter of the inserted stent. A variant of this model, the so-called CASP with intervention (CASPI), raises opportunity to remove the septic focus by a second operation according to common procedures in clinical practice. CASP is an easily learnable and highly reproducible model that closely mimics the clinical course of abdominal sepsis. It leads way to study on questions in several scientific fields e.g. immunology, infectiology, or surgery.

\section{Video Link}

The video component of this article can be found at https://www.jove.com/video/2299/

\section{Protocol}

\section{Preparation of the Mouse}

1. Anesthetize the mouse by intraperitoneal injection of the narcotic fluid (see table of reagents) and place it in supine position.

2. The feet of the mouse need to be fixed with tape on the plate to ensure a stable position of the animal during the operation.

\section{Operation}

1. Take an i.v. cannula, which is commonly used for i.v. injections in humans, and carve its plastic tube circularly $2 \mathrm{~mm}$ from its tip. We use three different sizes of cannulas to control mortality rates (BD Venflon 18GA (1,2x45mm, $80 \mathrm{~mL} / \mathrm{min}) ; 16 \mathrm{GA}(1,7 \times 45 \mathrm{~mm}, 180 \mathrm{~mL} / \mathrm{min}) 14 \mathrm{GA}$ $(2,0 \times 45 \mathrm{~mm}, 270 \mathrm{~mL} / \mathrm{min}))$.

2. After thorough disinfection of the abdominal skin, incise it along its midline about $15 \mathrm{~mm}$. Open the peritoneal cavern by incising the abdominal muscles and the peritoneum along the linea alba.

3. Identify the cecal pole and pull out carefully cecum, terminal ileum and ascending colon out of the abdomen by the use of cotton swabs.

4. $15 \mathrm{~mm}$ distal from the ileocecal valve, one has to pierce the wall of the ascending colon with a $7 / 0$ suture. Thereby, lesions of the gut vessels have to be avoided. The suture is fixed on the colonic wall by two surgical knots.

5. Now puncture the ascending colon with the prepared cannula 1-2 mm proximal from the $7 / 0$ suture. Carefully insert the cannula into the colon until the furrow in the plastic tube is on a level with the serosa.

6. Put the free ends of the $7 / 0$ suture around the cannula and place a two-fold knot exactly into the prepared furrow of the plastic tube.

7. Now, take the needle of the $7 / 0$ suture and stitch it through the antimesenteric wall of the colon. Consecutively, two surgical knots have to be performed to additionally fix the plastic tube in the colonic wall. Cut the suture ends.

8. Retrieve the iron part of the cannula a little and cut the plastic tube off close $(1 \mathrm{~mm})$ to the fixing $7 / 0$ suture.

9. Now one has to carefully milk stool from the cecum towards the colon stent by the use of cotton swabs until a small tip of stool appears on top of the stent.

10. Put the gut back into the peritoneal cavern and performe fluid resuscitation by intraperitoneal administration of $0.5 \mathrm{~mL}$ of saline solution.

11. Close the peritoneum with continuous suture $(4 / 0)$. 
12. Close the skin with singular sutures (4/0).

\section{Postoperative Care}

1. Put the animal back in its cage, which should contain enough food and water. For analgesia, intraperitoneal administration of a powerful analgesic substance (we use buprenorphine) should be regularly performed.

2. Within the first two days after the operation, one has to control the animals every 6 hours.

\section{Sham CASP}

1. Perform steps 1.1-2.4.

2. Do not puncture the colon. Just perform steps $2.6-2.8$.

3. Perform steps 2.10-3.2.

\section{CASPI}

1. Perform CASP using a $14 \mathrm{G}$ cannula following steps 1.1-3.1

2. $5 \mathrm{~h}$ after $\mathrm{CASP}$, one has to operate the animal again. Prepare and anesthetize the mouse again according to steps $1.1-1.2$

3. Open the sutures of the abdominal wall.

4. Pull out the ascending colon with the stent inserted.

5. Carefully cut the sutures fixing the stent and remove the stent.

6. Close the defect in the colon with single inverting sutures $(7 / 0)$.

7. Put the gut back into the abdominal cavity and flush the latter twice with $10 \mathrm{~mL}$ of saline solution.

8. Close the peritoneum with continuous suture (4/0).

9. Close the skin with singular sutures $(4 / 0)$.

10. Follow steps 3.1-3.2

\section{Representative Results}

Within a few hours following the operation, animals show clinical signs of a beginning sepsis. Typical symptoms of the disease are reduced mobility, horrent coat, sweating, decreased food intake, loss of weight and also reduced getaway behaviour. Animals developing a severe peritonitis with consecutive systemic infection normally die within $48 \mathrm{~h}$. Depending on the inserted stent size, distinct mortality rates can be generated. A $14 \mathrm{G}$ stent results in $100 \%$ mortality, $16 \mathrm{G}$ gives $70 \%$ mortality, and mortality rates of $50 \%$ can be reached by using an $18 \mathrm{G}$ stent (Figure 1a). The Sham operation results in a survival rate of $100 \%$. Usually, all animals die inbetween the first 48 hours after CASP. Thus, all animals that are alive at this time point can be regarded as survivors. However, we recommend an additional observation period of at least 72 hours to detect rare "late deaths" after CASP.

The outcome of CASPI depends on the period of time after CASP when the stent is removed. An intervention $3 \mathrm{~h}$ after CASP results in a survival rate of $45 \%$. If the stent is removed $5 \mathrm{~h}$ after CASP, only $10 \%$ of the animals survive the procedure. A stent removal after $9 \mathrm{~h}$ results in a mortality of $100 \%$ (Figure 1b). Sham CASPI leads to $100 \%$ survival. For CASPI settings, we recommend an observation period of 10 days because there usually are animals, which die later than 48 hours after CASP.

Macroscopic inspection of the abdominal situs 24 hours after CASP shows typical signs of peritonitis: edema, vasodilatation, erythematic gut wall, sharp demarcation of Peyer's patches, gut paralysis, free fluid, and septic secretion (Figure 2).

The systemic infection can be shown by bacteriological analysis. 12 hours after CASP, massive bacterial amounts can be detected in peritoneal lavage, blood, liver, lung, spleen, and kidneys. Qualitative analysis of bacteria reveals systemic infection by typical gut bacteria like $E$. coli, bacteroides species, enterococcus species, etc. (Figure 3).

CASP-induced sepsis manifests in local and also systemic release of pro- and anti-inflammatory cytokines and chemokines. 12 hours following CASP, significant amounts of TNF, IL-1 $\beta$, IL-6, IL-10, MCP-1 and others can be measured by ELISA in blood but also in supernatants of organ suspensions, e.g. liver, lung, spleen, kidney (Fig 4).

When establishing CASP in your laboratory, survival rates, bacteriology and cytokine release should be taken as control parameters for correct performance of CASP. Later on, these parameters can be taken as standard read out system for all experimental settings. 

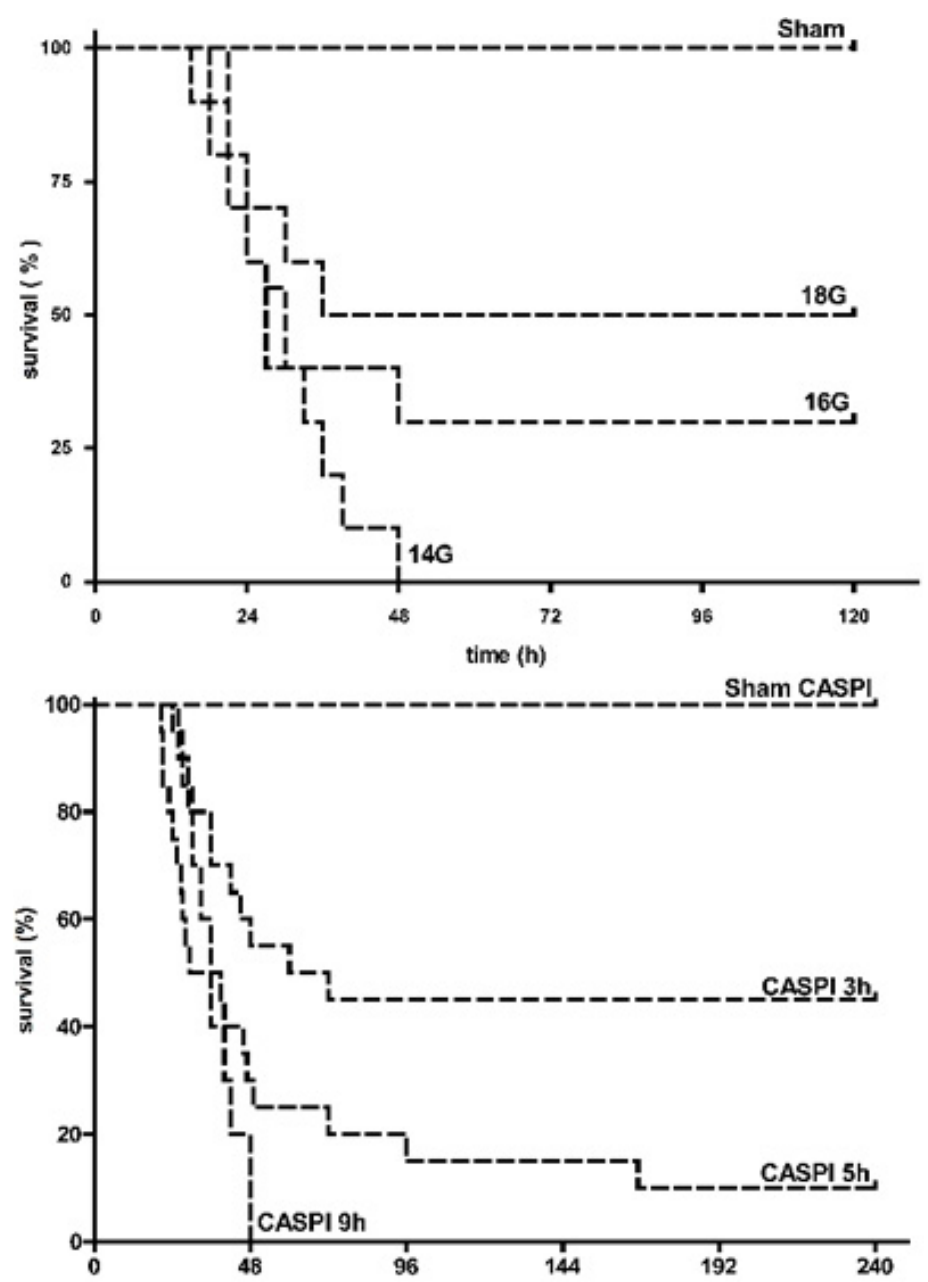

Figure 1a: Survival after CASP. Survival rates following CASP surgery depend on the diameter of the inserted stent. 14G CASP results in a mortality rate of $100 \%, 16 \mathrm{G}$ CASP results in $70 \%$ mortality, and $18 \mathrm{G}$ CASP leads to $50 \%$ mortality. Sham CASP is associated with a survival of $100 \%$. $n=20$ /group. B: Survival rates following CASPI surgery depend on the time of stent removal. Stent removal at $3 \mathrm{~h}$ after $14 \mathrm{G}$ CASP leads to a mortality rate of $55 \%$. Stent removal at $5 \mathrm{~h}$ after $14 \mathrm{G}$ CASP results in a mortality rate of $90 \%$ whereas stent removal at $9 \mathrm{~h}$ after $14 \mathrm{G}$ CASP results in $100 \%$ mortality. Sham CASPI (5h) results in a survival of 100\%. $n=10 /$ group (Sham CASPI; CASPI 9h), $n=20 / g r o u p ~(3 h$ CASPI; 5 h CASPI).
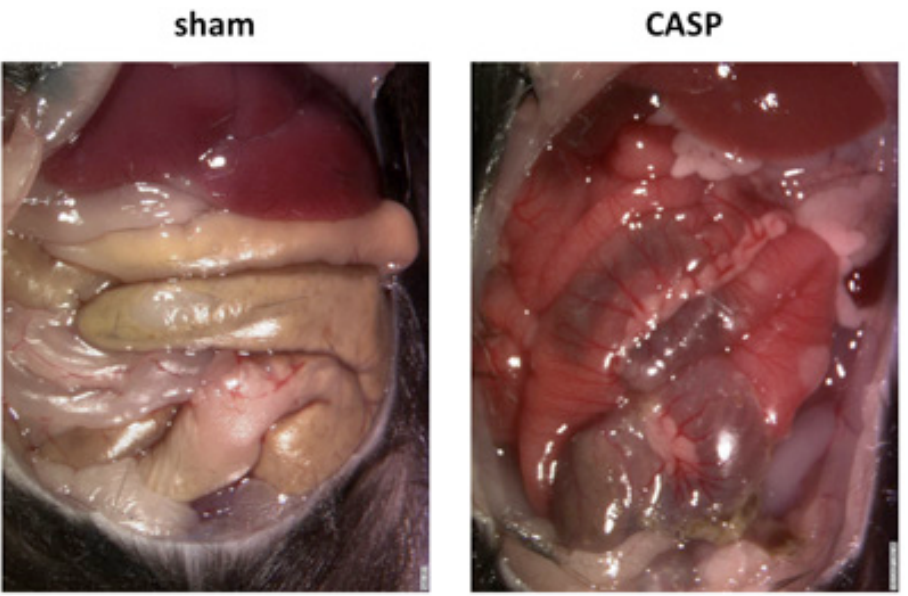

Figure 2: Abdominal situs 24h after CASP. The abdominal wall was removed 24h after 16G CASP or sham CASP, respectively. The situs of the sham operated mouse looks physiological. In contrast, CASP results in edema, hyperemia, dilatated bowels, and demarcation of Peyer's patches demonstrating severe peritonitis. 


\section{blood}

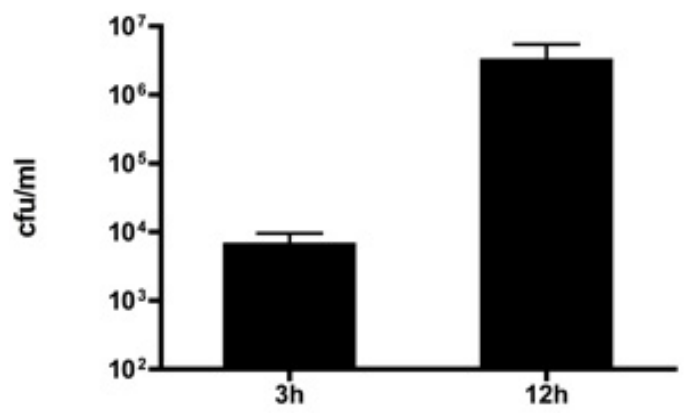

Figure 3: Bacteriology. CASP leads to severe infection of several compartments and organs by gut bacteria within $12 \mathrm{~h}$ after operation. Bacterial numbers are given as colony forming units (cfu) per $\mathrm{mL}$, respectively. Bacterial cultures taken from sham operated mice are completely sterile (not shown). 16G CASP, $n=5 /$ group.

\section{plasma}
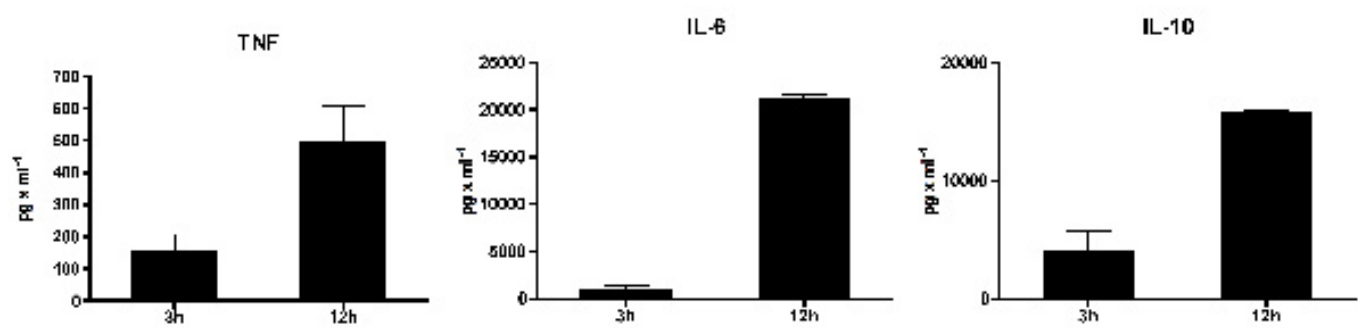

Figure 4: Cytokines. CASP triggers a systemic immune response as several cytokines and chemokines are detectable in plasma and also in organ supernatants. The figure exemplarily shows the pro-inflammatory cytokines tumor necrosis factor (TNF) and interleukin-6 (IL-6) as well as the predominantly anti-inflammatory IL-10. $12 \mathrm{~h}$ after CASP, remarkable levels can be detected in plasma, liver, lung, and spleen. However, IL-10 cannot be detected in lung and spleen after CASP. Sham operation does not lead to any cytokine secretion (not shown). 16G CASP, $n=5 / g r o u p$.

\section{Discussion}

CASP is a polymicrobial model of abdominal sepsis that meets most criteria postulated for a valuable sepsis model: CASP can be performed in small animals, is highly reproducible, easily to monitor by standard parameters, provides good read out possibilities, and closely mimics clinical settings of abdominal sepsis. In a comparative study we could show that CASP leads to diffuse peritonitis with early and steadily increasing systemic infection and inflammation (systemic inflammatory response syndrome), whereas cecal ligation and puncture (CLP) reveals a model of intra-abdominal abscess formation with sustained and minor signs of systemic inflammation (1). The technique of CASPI presents a possibility to simulate the clinical course of peritonitis with consecutive surgical focus sanitation, which is the most important therapeutic principle in humans. CASP can be applied for all questions around sepsis or peritonitis and is therefore useful for all disciplines dealing with sepsis research, for example immunology, pharmacology, surgery, intensive care medicine, etc. A selection of publications concerning different subjects of sepsis using the CASP model is given below (1-14).

When starting with CASP, one should be aware that some practice is necessary to perform the operation exactly and quickly. Surgical experience may be helpful but is not required. We recommend detection of letality as a parameter for correct performance of CASP. One should start with 14G CASP as a large stent is easier to handle than a thinner one. A trained CASP surgeon is able to operate one mouse within 10 minutes. Before starting any experiments, you should make sure that you are near that period. When you gained some experience with the surgical procedure, you should conduct a survival kinetic to check your skills. 14G CASP should result in a $100 \%$ lethality. Beginners in our laboratory have to prove that they are able to generate a $100 \%$ lethality with $14 \mathrm{G}$ CASP before they start with experiments. Additionally, bacteriology and cytokine measurements should be performed to verify sepsis. If you have problems in the beginning with the microsurgical steps of CASP, the use of magnifying glasses or a surgical microscope may be helpful. But in principal, CASP can be well performed without these utilities. For reproduction of our survival rates, it is absolutely necessary that the same materials, especially the stents (BD Venflon, see below), are used. Otherwise, substantial variations of the results are possible. Additionally, we suggest that there can be variations of the results depending on, for example, the individuality of the surgeon. However, the influence of factors like that should be small.

In summary, CASP is a practicable, simple, reproducible and valuable sepsis model that mimics closely the clinical situation of abdominal sepsis. It is therefore suitable for all fields of sepsis research.

\section{Disclosures}

No conflicts of interest declared. 


\section{Acknowledgements}

The authors thank Nico Zantl, Klaus Pfeffer, and Bernhard Holzmann. This study was supported by the Deutsche Forschungsgemeinschaft, Bonn-Bad Godesberg, Germany (GRK-840; Vo 450/10-1).

\section{References}

1. Maier, S., Traeger, T., Entleutner, M., Westerholt, A., Kleist, B., Huser, N., Holzmann, B., Stier, A., Pfeffer, K. \& Heidecke, C.D. Cecal ligation and puncture versus colon ascendens stent peritonitis: two distinct animal models for polymicrobial sepsis. Shock 21, 505-511 (2004).

2. Bougaki, M., Searles, R.J., Kida, K., De Yu, J., Buys, E.S. \& Ichinose, F. NOS3 protects against systemic inflammation and myocardial dysfunction in murine polymicrobial sepsis. Shock (2009).

3. Buras, J.A., Holzmann, B. \& Sitkovsky, M. Animal models of sepsis: setting the stage. Nat Rev Drug Discov 4, $854-865$ (2005).

4. Busse, M., Traeger, T., Potschke, C., Billing, A., Dummer, A., Friebe, E., Kiank, C., Grunwald, U., Jack, R.S., Schutt, C., Heidecke, C.D., Maier, S. \& Broker, B.M. Detrimental role for CD4+ T lymphocytes in murine diffuse peritonitis due to inhibition of local bacterial elimination. Gut 57, 188-195 (2008).

5. Daubeuf, B., Mathison, J., Spiller, S., Hugues, S., Herren, S., Ferlin, W., Kosco-Vilbois, M., Wagner, H., Kirschning, C.J., Ulevitch, R. \& Elson, G. TLR4/MD-2 monoclonal antibody therapy affords protection in experimental models of septic shock. J Immunol 179, 6107-6114 (2007).

6. Entleutner, M., Traeger, T., Westerholt, A., Holzmann, B., Stier, A., Pfeffer, K., Maier, S. \& Heidecke, C.D. Impact of interleukin-12, oxidative burst, and iNOS on the survival of murine fecal peritonitis. Int J Colorectal Dis 21, 64-70 (2006).

7. Kerschen, E.J., Fernandez, J.A., Cooley, B.C., Yang, X.V., Sood, R., Mosnier, L.O., Castellino, F.J., Mackman, N., Griffin, J.H. \& Weiler, H. Endotoxemia and sepsis mortality reduction by non-anticoagulant activated protein C. J Exp Med 204, 2439-2448 (2007).

8. Kiank, C., Koerner, P., Kessler, W., Traeger, T., Maier, S., Heidecke, C.D. \& Schuett, C. Seasonal variations in inflammatory responses to sepsis and stress in mice. Crit Care Med 35, 2352-2358 (2007).

9. Lustig, M.K., Bac, V.H., Pavlovic, D., Maier, S., Grundling, M., Grisk, O., Wendt, M., Heidecke, C.D. \& Lehmann, C. Colon ascendens stent peritonitis--a model of sepsis adopted to the rat: physiological, microcirculatory and laboratory changes. Shock 28, 59-64 (2007).

10. Traeger, T., Kessler, W., Assfalg, V., Cziupka, K., Koerner, P., Dassow, C., Breitbach, K., Mikulcak, M., Steinmetz, I., Pfeffer, K., Heidecke, C.D. \& Maier, S. Detrimental role of CC chemokine receptor 4 in murine polymicrobial sepsis. Infect Immun 76, 5285-5293 (2008).

11. Traeger, T., Kessler, W., Hilpert, A., Mikulcak, M., Entleutner, M., Koerner, P., Westerholt, A., Cziupka, K., van Rooijen, N., Heidecke, C.D. \& Maier, S. Selective depletion of alveolar macrophages in polymicrobial sepsis increases lung injury, bacterial load and mortality but does not affect cytokine release. Respiration 77, 203-213 (2009).

12. Traeger, T., Mikulcak, M., Eipel, C., Abshagen, K., Diedrich, S., Heidecke, C.D., Maier, S. \& Vollmar, B. Kupffer cell depletion reduces hepatic inflammation and apoptosis but decreases survival in abdominal sepsis. Eur J Gastroenterol Hepatol Epub ahead of print (2010).

13. Weighardt, H., Kaiser-Moore, S., Schlautkotter, S., Rossmann-Bloeck, T., Schleicher, U., Bogdan, C. \& Holzmann, B. Type I IFN modulates host defense and late hyperinflammation in septic peritonitis. J Immunol 177, 5623-5630 (2006).

14. Zantl, N., Uebe, A., Neumann, B., Wagner, H., Siewert, J.R., Holzmann, B., Heidecke, C.D. \& Pfeffer, K. Essential role of gamma interferon in survival of colon ascendens stent peritonitis, a novel murine model of abdominal sepsis. Infect Immun 66, 2300-2309 (1998). 